5th IFAC International Conference on Fieldbus Systems and their Applications

Nov, 14-15 2005

Puebla, Mexico

http://www.fet2005.cs.buap.mx

\title{
DEPENDABILITY EVALUATION OF REAL-TIME APPLICATIONS DISTRIBUTED ON TDMA- BASED NETWORKS
}

\author{
François Simonot ${ }^{1}$, Françoise Simonot-Lion ${ }^{2}$, YeQiong Song ${ }^{2}$ \\ (1) IECN - ESSTIN, (2) LORIA - INPL - UHP Nancy 1 \\ Campus Scientifique, BP 239-54506 Vandouvre-lès-Nancy (France) \\ simonot@esstin.uhp-nancy.fr ; \{simonot,song\}@loria.fr
}

\begin{abstract}
TDMA is largely adopted by many networks for providing to real-time applications with determinism. However, this determinism could be jeopardized under the transient environmental perturbations, causing application failures. In this paper, we investigate the impact of the transient perturbations (especially due to Electromagnetic Interferences) on the application dependability. We give a contribution on the method for evaluating the application failure probability in function of the TDMA cycle error probability within an EMI zone. This method extends the existing one on the "consecutive-k-out-of-n:F" systems to including variable probability. Numerical applications have shown its efficiency for computing the application failure probability within a given EMI area. Copyright (C) 2005 IFAC
\end{abstract}

Keywords: dependability, reliability, TDMA, TTP/C, FlexRay, EMI perturbation, transient fault.

\section{INTRODUCTION}

For achieving deterministic communication properties, many real-time networks use TDMAbased protocols. Such a protocol eases the design of time-triggered applications. TDMA slots are assigned to each data producers in a periodic (or cyclic) way with a fixed TDMA cycle duration. A receiver node periodically receives therefore data it consumes at fixed TDMA time slots so that associated actions can be executed at the right time. Moreover an absence of data production or transfer can be easily detected by the system making the consumer to take a right decision if necessary. In practice, for providing more reliability, the designer of time-triggered applications generally makes data producers to send data to the consumer with period much smaller than necessary (i.e., Nyquist frequency), tolerating thus occasional production or transmission errors to some extend. This is particularly interesting for systems operating at harsh conditions (i.e., subjected to environmental perturbations) provoking transient errors.

For instance, when TDMA-based networks are subjected to EMI (Electromagnetic Interference) perturbations, which are, for example, the typical case of automotive networks, message transmissions can be erroneous. In most of networks such as LIN, $\mathrm{TTP} / \mathrm{C}$ and FlexRay, transmission errors are detected but erroneous messages are not systematically retransmitted.

In this paper we focus on the analysis of the dependability of a TDMA-based network faced to EMI perturbations with respect to the dependability properties required by a safety critical application distributed on this network. In particular, examples of such an application are in-vehicle embedded systems. With the increasing deployment of electronic embedded components in vehicles, one hears in the media more and more vehicle failures due to the malfunctioning of electronic components.

If today's identified problems find rapidly their solutions, some non-identified problems, making random behaviours of the vehicle, remains unsolved (e.g. vehicle speed blocked by the cruise control system). It seems that EMI perturbations are the main cause of those random behaviours of the vehicle. The Wall Street Journal, edited 8 September 1997, quoted in Risk Digest, (1997) talked about accidents involving vehicles, which to all appearances were caused by EMI. More recently in France, it happens that the cruise control system of certain cars behaves randomly (e.g. impossible to reduce the speed!). 


\section{TDMA cycle (length $=T)$}

Control law execution

(activation period $=T$ )

Drivers' request really transmitted on the network
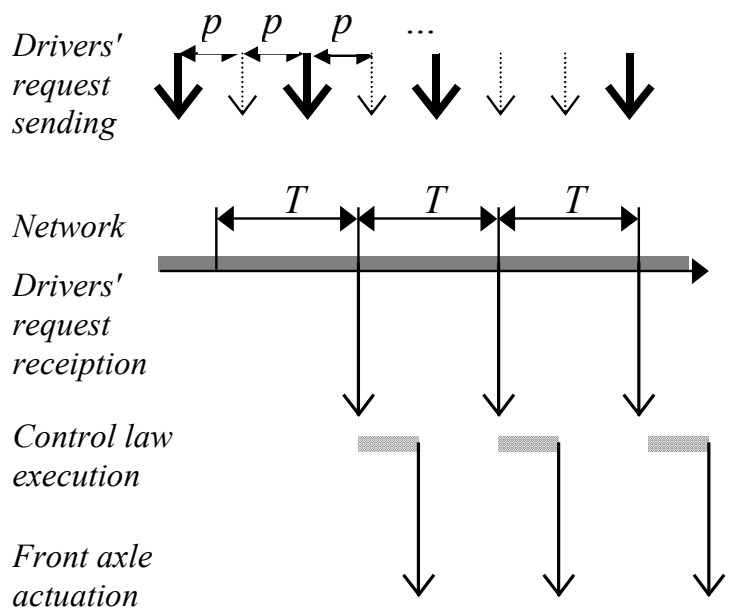

Fig. 1 - Drivers' request transmission

This problem can become even more critical with future integration of X-by-wire systems in a vehicle. So the behaviour of such a system face to EMI has to be evaluated and in particular the impact of the network on the vehicle dependability has to be analysed.

Let us take as an example a Steer-by-Wire system. Critical functions such as front axle control are assumed to be executed on a micro controller (ECU - Electronic Control Unit) periodically taking into account the output of the physical system to control, environmental information and the driver request given at the hand wheel level. This last information is produced periodically by another ECU and transmitted through a TDMA-based network to control law (Fig. 1). Obviously, for this application, the length of the TDMA cycle is equal to the activation period of the control law ( $T$ in Fig. 1)). The lack of this drivers' request, due for example to transmission errors, during a short-term, that is for a limited number of control law consecutive executions, can be tolerated. However, it is obvious that long-term absence of input data at the consumer side can lead to dangerous situations. So it is important to be able to evaluate the risk that consecutive erroneous TDMA cycles exceed the application-tolerating threshold (in terms of a given TDMA cycle number or equivalently the time length). This risk is termed in this paper the application failure probability, which gives a metric to measure the application robustness. Obviously this probability will depend on the perturbation model.

In this paper, we propose a method for the evaluation of the application failure probability for critical systems distributed on a TDMA-based network and subject to several profiles of EMI perturbations. This evaluation is based on the assumption that one single non-erroneous cycle is enough for bringing the system to its normal state. This hypothesis is verified for the given Steer-by-wire architecture that we use as example in the following.

However, only little work has been done to deal with the application failure probability under EMI perturbations and more generally under transient faults. In (Navet et al., 2000) we first addressed the problem for applications distributed over CAN and introduced the worst-case deadline failure probability as one of the possible application dependability metrics. In (Broster at al., 2004), the impact of the EMI on the real-time delivery capability of CAN and TTCAN has been evaluated. These results cannot be readily applied to the TDMA-based networks. In fact, CAN retransmits whenever a transmission error occurs whereas in TDMA-based networks, there is no systematic retransmission upon transmission errors.

In our previous work (see Wilwert et al., 2003 and Wilwert et al., 2005), we focused either on the evaluation of the X-by-wire application-tolerating threshold or the method to evaluate the application failure probability under restrictive hypothesis on the perturbation model. For instance, the probability for a TDMA cycle to be erroneous, termed in the following "TDMA cycle error probability", has been assumed constant. In fact it is not the general case. A given source can cause EMI that varies in time and / or the distance between a perturbation source and the vehicle can vary in time. It is shown in (Chao et al., 1995) that the evaluation of the application failure probability with constant TDMA cycle error probability can be performed based on the classic results on the "consecutive-k-out-of-n: $F^{\prime \prime}$ systems. In this paper, we extend these results by dealing with the application failure probability evaluation with variable TDMA cycle error probability. So the previous theoretic results on the "consecutive-k-outof $n: F^{\prime \prime}$ systems have to be extended.

In what follows, we will give in section 2 a description of the EMI perturbations that an invehicle application can meet; such perturbations may provoke TDMA cycle errors (because of either transmission errors or producing errors). Section 3 is devoted to our main contribution that is a method for evaluating the application failure probability with variable TDMA cycle error probability. Section 4 shows numerical applications for some typical error models (TDMA cycle error probability profiles) and that are obtained on a Steer-by-wire system. Section 5 gives the concluding remarks.

\section{EMI PERTURBATIONS AND ERROR MODELS}

Electromagnetic interferences are mainly caused by radio-communication transmitters, radars, and high voltage lines. Their influence on electronic components depends on the frequency and strength of the electromagnetic fields. In automotive industry, each carmaker specifies an internal regulatory policy that imposes the robustness level of electronic device 


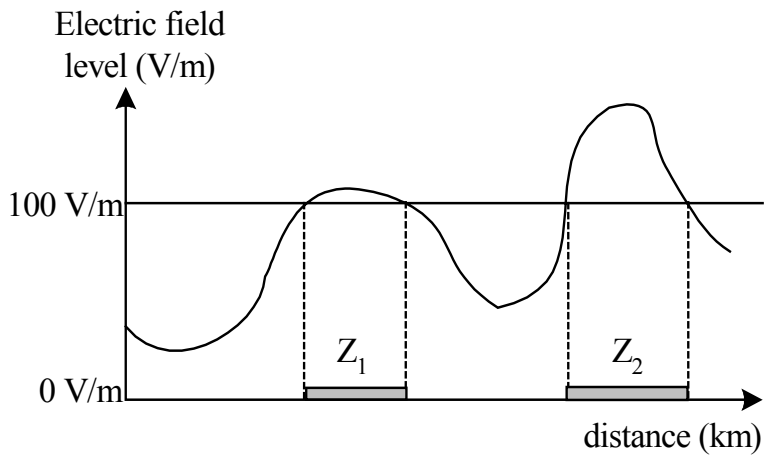

Fig. 2 Example of electric field level of a reference road

with electromagnetic interference sources under a given voltage level and for a given interval of frequencies. So a test process is applied on each electronic component in order to verify its conformity to the specific carmaker standard. Nevertheless, this conformity is just proved for given frequencies and voltage level. In fact, it is established that the testing condition are not met everywhere; it exists some traffic areas, for example near airports, where a vehicle can go through an area subject to a higher level of voltage and / or other frequencies than the specified ones and therefore, the probability that an in-vehicle embedded system can be corrupted by electromagnetic interferences is not zero. For example, carmakers often consider that the upper limit for the robustness assessment of electronic components is $100 \mathrm{~V} / \mathrm{m}$. This is to say that when a car goes through an EMI perturbed zone with a force higher than 100 volts per meter, its embedded electronic systems may exhibit errors.

Some sources of electromagnetic interferences are statically disposed along the road (for example, radars or high voltage lines). CEERF, a French project, funded by Ministry of Transport, proposed a characterization of the electromagnetic pollution for the French road system (Predit-CEERF, 2003). This project targeted mainly the automotive industry by proposing a cartography of the EMI sources and electromagnetic field levels in France and a method for its updating. These results are obtained thanks to a monitor embedded in a car and whose role is to record the frequency and the level of the ambient electromagnetic field during a journey along several representative roads. From this recording, we are able to select the length (in $\mathrm{km}$ ) of each area under EMI perturbation of higher than $100 \mathrm{~V} / \mathrm{m}$ (see Fig. 2 ); on the represented trajectory, two parts of this trajectory, areas $Z_{1}$ and $Z_{2}$, are subject to perturbations of more than $100 \mathrm{~V} / \mathrm{m}$ ).

Without losing generality, in what follows, we will only focus on the analysis within one EMI zone. In fact, as assumed in section 1, for the targeted application, one single correct reception of the input data at the consumer side before the applicationtolerating threshold is required for bringing the

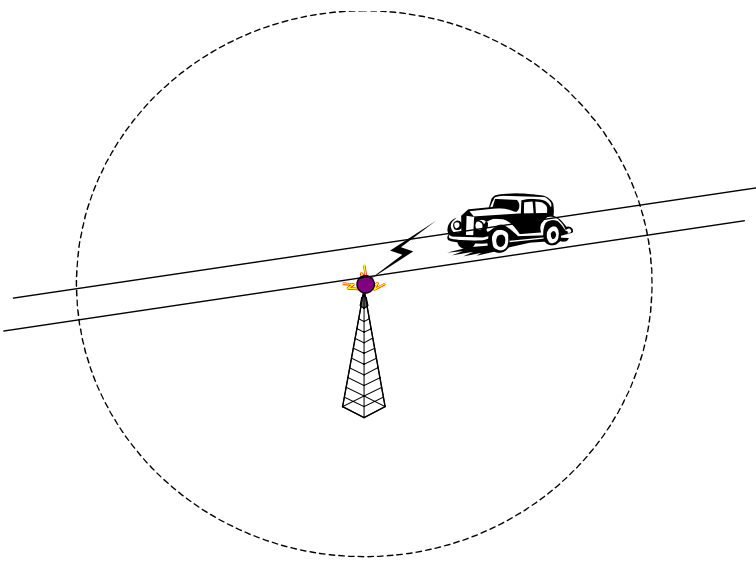

Fig. 3 Example of an EMI zone with variable perturbation levels

system to its normal state. So multiple zones (as they are independent each other) can be treated separately. In (Wilwert et al., 2005), we proposed how to evaluate the failure probability for a specific vehicle trajectory such as what is shown in Fig. 2.

For passing through a given EMI area, a vehicle (assumed with a constant speed) will take a certain time called hereafter the passing through time and denoted by $T_{z}$. If one knows the TDMA cycle duration $T_{c y c}$, the passing through time can also be represented in terms of the number of TDMA cycles $n$. In (Wilwert et al., 2003) and (Wilwert et al., 2005), for taking into account the worst-case protocol recovery overhead, we evaluated this value:

$$
n=\left\lceil\frac{T_{z}}{T_{c y c}}\right\rceil+2
$$

We assume furthermore that each TDMA cycle has a non-null error probability within an EMI perturbation area. In general this probability is variable and depends on the position of the vehicle within the zone. Intuitively the stronger the electric field (measured in $\mathrm{V} / \mathrm{m}$ ) is, the higher the probability to have an erroneous TDMA cycle is. Fig. 3 shows a typical situation where a vehicle passes through an EMI area generated by a radio transmitter. If a rough approximation by the free space propagation model (Rappaport, 1995) can be used, the electric field strength at a point will be inversely proportional to the square of its distance to the source.

Unfortunately, the exact characterization of the TDMA cycle error probabilities within a zone has not been realized because of large measurements data needed for being statistically confident.

In this study, to get a general idea about the impact of EMI on the application robustness, we will evaluate the application failure probability by analysing some typical profiles of $P=\left(p_{1}, p_{2}, \ldots, p_{n}\right)$ called hereafter "error models". 


\section{$2.1 \quad$ Constant-P model}

This first error model describes a constant perturbation. For the total passing through time of $n$ TDMA cycles of a given EMI zone, we assume that each TDMA cycle has a same error probability, i.e. $p_{i}=p$ for all $i=1,2, \ldots, n$.

Of course, this may not correspond to an actual situation. However, it is often the case of the laboratory tests. We keep it as a reference model for further comparisons.

\section{$2.2 \quad$ Radio-P model}

The second error model is a Radio- $P$ model. It is designed to represent the error model of a vehicle passing through an EMI area of $n$ TDMA cycles generated by a radio transmitter (e.g. Fig. 3). Assuming that the free space propagation model (Rappaport, 1997) is adopted and the error probability of a TDMA cycle is somehow proportional $^{1}$ to the received electric field strength, for a given TMDA cycle $i(i=1,2, \ldots, n)$ we give its error probability by:

$$
p_{i}=\frac{a}{\left(\frac{n+1}{2}-i\right)^{2}+b}
$$

Where $a$ and $b$ (with $a \leq b$ ) are free parameters which can be adjusted for fitting to a concrete situation. This model is illustrated in Fig. 4.

\section{$2.3 \quad$ Radar-P model}

The third error model is a Radar-P model. It is proposed to represent the error model of a vehicle passing through an EMI zone of $n$ TDMA cycles generated by a radar source (e.g. near to an airport). The electric field varies periodically with the radarscanning period of $T$ (with $T<T_{z}$ ). Again we assume that the error probability of a TDMA cycle is proportional to the received electric field strength.

For a given TMDA cycle $i(i=1,2, \ldots, n)$ we give its error probability by:

$$
p_{i}=a+b \sin \frac{2 \pi}{T} i
$$

Where $a$ and $b$ (with $a-b>0$ and $a+b \leq 1$ ) are free parameters which can be adjusted for fitting to a concrete situation. Fig.4 depicts the trends of a Radar-P model.

1 This is only a general assumption. In practice, node and communication channel redundancy is often used to reduce the impact of EMI perturbations. The error probability estimation for a given perturbation could be more complex and field tests are necessary. In (Wilwert et al., 2005), we discussed in more detail the ways to estimate it. $f_{\text {Radio }-P}(i)=\frac{10}{\left(\frac{n+1}{2}-i\right)^{2}+40} \quad f_{\text {Radar }-P}(i)=0,1+0,09 \sin \frac{2 \pi}{375}$

TDMA cycle

error probability

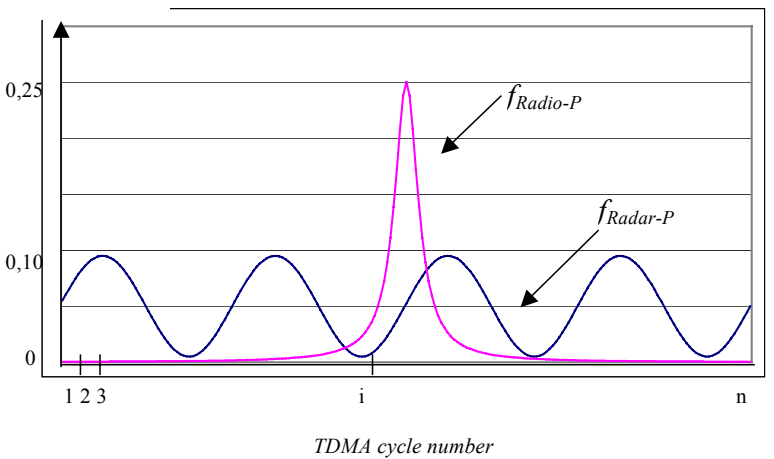

Fig. 4 Trends of Radio- $P$ and Radar- $P$ models

\section{APPLICATION FAILURE PROBABILITY EVALUATION}

\subsection{Existing results for Constant-P model}

Knowing a zone of $n$ TDMA cycles and the application-tolerating threshold of $k$ TDMA cycles, for constant $p$, the problem can be treated using existing results on the reliability of a system composed of an ordered sequence of $n$ components and such that the system fails if and only if at least $k$ consecutive components fail.

This kind of systems are termed "consecutive-k-out$o f-n: F^{\prime \prime}$ systems and denoted by $C(k, n: F)$. For such a system, we note $n$ the number of components, $p$ the probability that a component fails, $L_{n}$ a number of consecutive failed components and $k-1$ the largest tolerable number of consecutive failed components; the reliability of the system is evaluated by the probability that $L_{n}<k$, denoted by $P\left(L_{n}<k\right)=R(k, n ; p)$, or equivalently the failure probability $\quad P_{\text {fail }}=P\left(L_{n} \geq k\right)=1-R(k, n ; p)$. The following formula was proposed first in (Burr and Cane, 1961) and then simplified in (Lambris and Papastavridis, 1985) and (Hwang, 1986).

$$
R(k, n ; p)=\sum_{m=0}^{\lfloor(n+1) /(k+1)\rfloor}(-1)^{m} p^{m k} q^{m-1}\left(\left(\begin{array}{c}
n-m k \\
m-1
\end{array}\right)+q\left(\begin{array}{c}
n-m k \\
m
\end{array}\right)\right)
$$

where $q=1-p$.

The numerical evaluation of $R(k, n ; p)$ via this formula is quite complex; so, we have developed in (Wilwert et al., 2005) a recurrent relation permitting to compute the failure probability for any $n$ and $k$.

For $P=\left(p_{1}, p_{2}, \ldots, p_{n}\right)$, as there does not exist closed form solutions, we give in the sequel a solution for $R(k, n ; P)$. 
3.2 New results for $P=(p 1, p 2, \ldots, p n)$ with non constant pi

Now this $P$, without loss of generality, let us consider an infinite sequence of independent Bernoulli trials $X_{1}, X_{2}, \ldots X_{n}, \ldots$ defined on the probability space $(\Omega, A, P)$ with $p_{i}=P\left(X_{i}=1\right)$ for $i \geq 1$.

We call "word" a sequence of consecutive successes of Bernoulli trials (when $X_{i}=1$ ).

The goal of our work is to investigate the probability law of the random variable $L_{n}$, the length of the longest word known during the $n$ first trials. To our great surprise, it seems that this problem, in case of the non-identically distributed random variables, has never been addressed.

We define:

- $T_{k}=$ the first instant where a sequence of $\mathrm{k}$ consecutive successes appear.

- $u_{n}(k)=P\left(L_{n}<k\right)$ for $n \geq 0$ and $k \geq 1$. For fixed $k \geq 1$, the sequence $u_{n}(k)=P\left(L_{n}<k\right)$ is decreasing and lower bounded by 0 .

- $\quad \lambda_{n}(k)=q_{n-k} p_{n-k+1} p_{n-k+2} \ldots p_{n}$ for $n \geq k$ with $q_{0}=1$ and $q_{n}=1-p_{n}$ if $n \geq 1$.

\section{Property1}

The sequence $u_{n}(k)=P\left(L_{n}<k\right)$ verifies the following relation:

For $k \geq 1$ and $n \geq k+1$,

$$
u_{n}(k)=u_{n-1}(k)-\lambda_{n}(k) u_{n-k-1}(k)
$$

with initial conditions :

$u_{n}(k)=1$ for $0 \leq n \leq k-1$ and $u_{k}(k)=1-\lambda_{k}(k)$.

Proof :

We have :

$$
P\left(T_{k}=n\right)=p_{n} p_{n-1} \ldots p_{n-k+1} q_{n-k} P\left(L_{n-k-1}<k\right)
$$

and

$$
P\left(L_{n} \geq k\right)=P\left(T_{k} \leq n\right),
$$

which give the following relation :

$u_{n}(k)=u_{n-1}(k)-\lambda_{n}(k) u_{n-k-1}(k) \quad$ for $\quad k \geq 1 \quad$ and $n \geq k+1$.

\section{End of proof}

Propertyl contains complete information on the behavior of $u_{n}(k)=P\left(L_{n}<k\right)$ and allows an exact calculation of the probability law of $L_{n}$ and provides an efficient algorithm for computing $u_{n}(k)$. Moreover, the following useful monotonic property of $u_{n}(k)$ can be established.

\begin{tabular}{|c|c|c|c|}
\hline $\begin{array}{l}\text { TDMA } \\
\text { Cycle } \\
\text { Length }\end{array}$ & $\begin{array}{l}\text { Application } \\
\text { failure }\end{array}$ & $\begin{array}{l}\begin{array}{l}\text { Number } \\
\text { of } \\
\text { TDMA }\end{array} \\
\text { Cycles } \\
\mathrm{N} \\
\end{array}$ & $\begin{array}{l}\text { Maximum } \\
\text { tolerable } \\
\text { number of } \\
\text { consecutive } \\
\text { erroneous } \\
\text { cycles } \\
\mathrm{k}\end{array}$ \\
\hline 4 & $3.30 \mathrm{E}-09$ & 377 & 10 \\
\hline 4.25 & $3.12 \mathrm{E}-08$ & 355 & 9 \\
\hline 4.5 & $2.95 \mathrm{E}-07$ & 336 & 8 \\
\hline 4.75 & 2.79E-07 & 318 & 8 \\
\hline 5 & $2.65 \mathrm{E}-07$ & 302 & 8 \\
\hline 5.25 & $2.53 \mathrm{E}-06$ & 288 & 7 \\
\hline 5.5 & $2.41 \mathrm{E}-06$ & 275 & 7 \\
\hline 5.75 & $2.31 \mathrm{E}-05$ & 263 & 6 \\
\hline 6 & $2.21 \mathrm{E}-05$ & 252 & 6 \\
\hline 6.25 & $2.12 \mathrm{E}-05$ & 242 & 6 \\
\hline 6.5 & $2.04 \mathrm{E}-05$ & 233 & 6 \\
\hline 6.75 & $1.98 \mathrm{E}-04$ & 225 & 5 \\
\hline 7 & $1.91 \mathrm{E}-04$ & 217 & 5 \\
\hline 7.25 & $1.84 \mathrm{E}-04$ & 209 & 5 \\
\hline 7.5 & $1.77 \mathrm{E}-04$ & 202 & 5 \\
\hline 7.75 & $1.72 \mathrm{E}-04$ & 196 & 5 \\
\hline 8 & $1.67 \mathrm{E}-04$ & 190 & 5 \\
\hline 8.25 & 0.00161977 & 184 & 4 \\
\hline 8.5 & 0.00157484 & 179 & 4 \\
\hline 8.75 & 0.0015299 & 174 & 4 \\
\hline 9 & 0.00148497 & 169 & 4 \\
\hline 9.25 & 0.00144902 & 165 & 4 \\
\hline 9.5 & 0.00140408 & 160 & 4 \\
\hline 9.75 & 0.00136813 & 156 & 4 \\
\hline 10 & 0.00133218 & 152 & 4 \\
\hline
\end{tabular}

Table 1 Application failure probability under Constant- $P$ error model

\section{Property2}

If $p_{j}^{\prime} \geq p_{j}$ for all $j \geq 1$, then $u_{n}(k) \geq u_{n}^{\prime}(k)$ for all $n$ and all $k \geq 1$.

That is to say: $p_{j}^{\prime} \geq p_{j}$ for all $j \geq 1$ implies that $L_{n}$ is stochastically less or equal to $L_{n}^{\prime}$ for all $n$.

It turns out that $p_{j}^{\prime} \geq p_{j} \geq p_{j}^{\prime \prime}$ for all $j \geq 1$ implies $u_{n}^{\prime \prime}(k) \geq u_{n}(k) \geq u_{n}^{\prime}(k)$ for all $n$ and all $k \geq 1$. 


\section{NUMERICAL RESULTS}

In this section we will apply the previously established algorithms (the complexity of the program varies with $n$ ) to the three typical error models called Constant- $P$, Radio- $P$ and Radar- $P$ described above.

We focus on an EMI perturbation with passing through time $T_{z}=1500 \mathrm{~ms}$ and application-tolerating threshold $T_{\max }=40 \mathrm{~ms}$. Note that, for the Steer-bywire system that we presented formerly, these values are extracted on the one hand, from the cartography of EMI sources and electromagnetic field levels in France (Predit-CERF, 2003) and, on the other hand, by executing a Matlab / Simulink model that integrates the control law, the physical system and the vehicle characteristics (Wilwert et al., 2005).

For a given TDMA cycle duration $T_{c y c}, n$ is given by equation 1, whereas the application-tolerating threshold in terms of the number of TMDA cycles, $k$, is given by:

$$
k=\left\lfloor\frac{T_{\max }}{T_{c y c}}\right\rfloor
$$

Our objective is to evaluate the application failure probability $P_{\text {fail }}$ for a given TDMA cycle duration $T_{c y c}$.

In order to also analyze the influence of the TDMA cycle duration on $P_{\text {fail }}$, we make vary $T_{c y c}$ and, consequently, the activation period of the control law from $4 \mathrm{~ms}$ to $10 \mathrm{~ms}$ with step of $0.25 \mathrm{~ms}$ (these values have to be specified both by automatic control specialists and by system architect designer). The obtained results provide guidelines for system designer to correctly dimensioning $T_{c y c}$ for meeting a specific requirement on $P_{\text {fail }}$.

\section{$4.1 \quad$ Constant-P model}

Let $p=0.1$, by using the algorithm given by property1, we get the following failure probability $P_{\text {fail }}$ (Table 1).

In view of the equations 1 and 5, when $T_{c y c}$ increases, $n$ and $k$ both decrease. As was seen in section 3.2, for a fixed $k$ value, the failure probability is an increasing function of $n$.

\section{$4.2 \quad$ Radio-P model}

Let $a=10$ and $11, b=20$ and 19 respectively, $p_{i}$ are given according to equation 2 . The failure probability $P_{\text {fail }}$ for different $T_{c y c}$ is given in Table 2 .

In addition to the general comments we have already made for the Constant- $P$ case, we can also observe the effect of property 2 .
Table 2 Application failure probability under Radio-

\section{$P$ error model}

\begin{tabular}{|c|c|c|c|c|}
\hline $\begin{array}{l}\text { TDMA } \\
\text { Cycle } \\
\text { Length }\end{array}$ & $\begin{array}{l}\text { Application } \\
\text { failure } \\
(a=10, b=20)\end{array}$ & $\begin{array}{l}\text { Application } \\
\text { failure } \\
(a=11, b=19)\end{array}$ & $\begin{array}{l}\text { Number } \\
\text { of } \\
\text { TDMA } \\
\text { Cycles }\end{array}$ & \\
\hline $\mathrm{T}_{\mathrm{cyc}}$ & $P_{\text {fail }}$ & $\mathrm{P}_{\text {fail }}$ & $\mathrm{n}$ & $\mathrm{k}$ \\
\hline 4 & $2.22 \mathrm{E}-08$ & 8.19E-08 & 377 & 10 \\
\hline 4.25 & 2.94E-07 & $9.73 \mathrm{E}-07$ & 355 & 9 \\
\hline 4.5 & $3.30 \mathrm{E}-06$ & $9.82 \mathrm{E}-06$ & 336 & 8 \\
\hline 4.75 & $3.30 \mathrm{E}-06$ & $9.82 \mathrm{E}-06$ & 318 & 8 \\
\hline 5 & $3.30 \mathrm{E}-06$ & $9.82 \mathrm{E}-06$ & 302 & 8 \\
\hline 5.25 & $3.12 \mathrm{E}-05$ & $8.32 \mathrm{E}-05$ & 288 & 7 \\
\hline 5.5 & $3.12 \mathrm{E}-05$ & $8.32 \mathrm{E}-05$ & 275 & 7 \\
\hline 5.75 & $2.46 \mathrm{E}-04$ & $5.86 \mathrm{E}-04$ & 263 & 6 \\
\hline 6 & $2.46 \mathrm{E}-04$ & $5.86 \mathrm{E}-04$ & 252 & 6 \\
\hline 6.25 & $2.46 \mathrm{E}-04$ & $5.86 \mathrm{E}-04$ & 242 & 6 \\
\hline 6.5 & $2.46 \mathrm{E}-04$ & $5.86 \mathrm{E}-04$ & 233 & 6 \\
\hline 6.75 & 0.001609891 & 0.00340238 & 225 & 5 \\
\hline 7 & 0.001609891 & 0.00340238 & 217 & 5 \\
\hline 7.25 & 0.001609891 & 0.00340238 & 209 & 5 \\
\hline 7.5 & 0.001609891 & 0.00340238 & 202 & 5 \\
\hline 7.75 & 0.001609891 & 0.00340238 & 196 & 5 \\
\hline 8 & 0.001609891 & 0.00340238 & 190 & 5 \\
\hline 8.25 & 0.008690406 & 0.01621666 & 184 & 4 \\
\hline 8.5 & 0.008690406 & 0.01621666 & 179 & 4 \\
\hline 8.75 & 0.008690406 & 0.01621666 & 174 & 4 \\
\hline 9 & 0.008690406 & 0.01621666 & 169 & 4 \\
\hline 9.25 & 0.008690406 & 0.01621666 & 165 & 4 \\
\hline 9.5 & 0.008690406 & 0.01621666 & 160 & 4 \\
\hline 9.75 & 0.008690406 & 0.01621666 & 156 & 4 \\
\hline 10 & 0.008690406 & 0.01621666 & 152 & 4 \\
\hline
\end{tabular}

In fact, if we note:

$$
\begin{aligned}
& P=\left(p_{1}, p_{2}, \ldots, p_{n}\right) \text { for } a=10 \text { and } b=20, \\
& P^{\prime}=\left(p^{\prime}{ }_{1}, p_{2}{ }_{2}, \ldots, p_{n}{ }_{n}\right) \text { for } a=11 \text { and } b=19,
\end{aligned}
$$

according to equation 2 , it turns out that $p^{\prime}{ }_{i}>p_{i}$ for all $i=1,2, \ldots, n$. So it is not surprising that $P_{\text {fail }}^{\prime}>$ $P_{\text {fail }}$ in Table 2.

\subsection{Radar-P model}

$p_{i}$ are given according to equation 3 with $a=0.1, b=$ 0.09 and radar scanning period $T=375 \mathrm{~ms}$ (i.e. $4 T=$ $\left.T_{z}\right)$,

The failure probability $P_{\text {fail }}$ for different $T_{c y c}$ is given in Table 3. 
Table 3 Application failure probability under Radar$\underline{P \text { error model }}$

\begin{tabular}{|c|c|c|c|}
\hline $\begin{array}{l}\text { TDMA } \\
\text { Cycle } \\
\text { Length }\end{array}$ & $\begin{array}{l}\text { Application } \\
\text { failure }\end{array}$ & $\begin{array}{l}\text { Number } \\
\text { of } \\
\text { TDMA } \\
\text { Cycles }\end{array}$ & $\begin{array}{l}\text { Maximum } \\
\text { tolerable } \\
\text { number of } \\
\text { consecutive } \\
\text { erroneous } \\
\text { cycles } \\
\mathrm{k} \\
\end{array}$ \\
\hline 4 & $5.55 \mathrm{E}-07$ & 377 & 10 \\
\hline 4.25 & $2.93 \mathrm{E}-06$ & 355 & 9 \\
\hline 4.5 & $1.57 \mathrm{E}-05$ & 336 & 8 \\
\hline 4.75 & $1.47 \mathrm{E}-05$ & 318 & 8 \\
\hline 5 & $1.38 \mathrm{E}-05$ & 302 & 8 \\
\hline 5.25 & $7.53 \mathrm{E}-05$ & 288 & 7 \\
\hline 5.5 & $7.14 \mathrm{E}-05$ & 275 & 7 \\
\hline 5.75 & $3.92 \mathrm{E}-04$ & 263 & 6 \\
\hline 6 & $3.74 \mathrm{E}-04$ & 252 & 6 \\
\hline 6.25 & $3.57 \mathrm{E}-04$ & 242 & 6 \\
\hline 6.5 & $3.42 \mathrm{E}-04$ & 233 & 6 \\
\hline 6.75 & 0.00192067 & 225 & 5 \\
\hline 7 & 0.00184522 & 217 & 5 \\
\hline 7.25 & 0.0017695 & 209 & 5 \\
\hline 7.5 & 0.00170302 & 202 & 5 \\
\hline 7.75 & 0.00164584 & 196 & 5 \\
\hline 8 & 0.00158847 & 190 & 5 \\
\hline 8.25 & 0.00907411 & 184 & 4 \\
\hline 8.5 & 0.008806 & 179 & 4 \\
\hline 8.75 & 0.00853722 & 174 & 4 \\
\hline 9 & 0.00826772 & 169 & 4 \\
\hline 9.25 & 0.00805156 & 165 & 4 \\
\hline 9.5 & 0.0077806 & 160 & 4 \\
\hline 9.75 & 0.0075632 & 156 & 4 \\
\hline 10 & 0.00734519 & 152 & 4 \\
\hline
\end{tabular}

This kind of numerical results can be used to verify whether a given application, distributed on TDMAbased networks and under a known EMI zone, can still meet the dependability constraint in terms of failure probability. It can also provide to a designer with guidelines for correctly dimensioning $T_{c y c}$ for meeting a specific requirement on application failure probability.

\section{CONCLUSION}

In this paper we have investigated the impact of the EMI perturbations on the dependability of applications distributed around TDMA-base networks where we assumed that application failure occurs when consecutive erroneous TDMA cycles exceed a certain threshold. This problem is of prime importance, especially for automotive industry as on the one hand, the most adopted embedded networks such as LIN, CAN and FlexRay are based on TDMA, and on the other hand many embedded applications (e.g. X-by-Wire systems) have to meet stringent dependability constraints, this even under EMI perturbations. We contributed to the method for evaluating the application failure probability. For this, we have proposed an important theoretic result which extends the existing one on the "consecutive- $k$ out-of- $n: F^{\prime \prime}$ systems to including variable probability. Although we have only analyzed several typical error models, our method is still available whatever the profile of $p_{i}$ may be. These probabilities can be obtained in practice by measurements. This method can also be used to study the system dependability of any transient perturbations.

\section{REFERENCES}

Broster, I., A. Burns, and G. Rodríguez-Navas (2004). Comparing real-time communication under electromagnetic interference, In Proceedings of the $16^{\text {th }}$ IEEE Euromicro Conference on Real-Time Systems, pages 45-52, Catania, Italy, July 2004.

Burr, E. J. and G. Cane (1961). End-toEnd Arguments in system design. In Biometrika, Vol. 48, pp.461-465.

Chao, M.T., J-C. Fu and M-V. Koutras (1995). Survey of reliability studies of consecutive-k-out-of-n:F and related systems. In IEEE Transactions on reliability, 44(1): 120-127, march 1995.

Hwang, F.-K. (1986). Simplified reliabilities for consecutive-k-out-of-n:F systems. In Algebraic Discrete Methods, Vol. 7, pp. 258-264, 1986.

Lambris, M. and S. G. Papastavridis (1985). Exact reliability formulas for linear and circular consecutive-k-out-of-n:F systems. In IEEE Transactions on Reliability, Vol. 34, pp. 124-126, 1985.

Navet, N., Y-Q. Song, and F. Simonot (2000). Worst-case deadline failure probability in real-time applications distributed over CAN. In Journal of Systems Architecture, 46(7).

PREDIT-CEERF (2005). Caractérisation de l'environnement électromagnétique routier en France. Technical report (in French), 2003.

Rappaport, T.S. (1995). Wireless Communications: Principles and Practice, Prentice Hall, 1995.

The Risks Digest (1997). GM car acceleration due to EMI. http://catless.ncl.ac.uk/Risks/19.38.html, 19 (38).

Wilwert, C., F. Simonot-Lion, Y.Q. Song and F. Simonot (2005). Quantitative Evaluation of the Safety of Xby-Wire Architecture subject to EMI Perturbations. In proceedings $10^{\text {th }}$ IEEE Conference of Emerging Technology for Factory Automation, ETFA'2005, September 2005, Catania, Sicily, Italy.

Wilwert, C., Y.Q. Song, F. Simonot-Lion and T. Clément (2003). Evaluating Quality of Service and Behavioral Reliability of Steer-by-Wire Systems. In Proceedings $9^{\text {th }}$ IEEE Conference of Emerging Technology for Factory Automation, ETFA'2003, Lisbonne, Portugal. 
